\title{
Classification performance of carbon black-polymer composite vapor detector arrays as a function of array size and detector composition
}

Michael C. Burl, Brian C. Sisk, Thomas P. Vaid, Nathan Saul Lewis

Michael C. Burl, Brian C. Sisk, Thomas P. Vaid, Nathan Saul Lewis, "Classification performance of carbon black-polymer composite vapor detector arrays as a function of array size and detector composition," Proc. SPIE 4742, Detection and Remediation Technologies for Mines and Minelike Targets VII, (13 August 2002); doi: 10.1117/12.479125

SPIE. Event: AeroSense 2002, 2002, Orlando, FL, United States 


\title{
Classification Performance of Carbon Black-Polymer Composite Vapor Detector Arrays As a Function of Array Size and Detector Composition
}

\author{
Michael C. Burl, ${ }^{\dagger}$ Brian C. Sisk, Thomas P. Vaid, and Nathan S. Lewis ${ }^{\mathrm{a}}$ \\ ${ }^{a}$ Division of Chemistry and Chemical Engineering, Noyes Laboratory, 127-72 \\ California Institute of Technology, Pasadena, CA 91125
}

${ }^{\dagger}$ Jet Propulsion Laboratory, 4800 Oak Grove Blvd., Pasadena, CA 91125

\begin{abstract}
The vapor classification performance of arrays of conducting polymer composite vapor detectors has been evaluated as a function of the number and type of detectors in an array. Quantitative performance comparisons were facilitated by challenging a collection of detector arrays with vapor discrimination tasks that were sufficiently difficult that at least some of the arrays did not exhibit perfect classification ability for all of the tasks of interest. For nearly all of the discrimination tasks investigated in this work, classification performance either increased or did not significantly decrease as the number of chemically different detectors in the array increased. Any given subset of the full array of detectors, selected because it yielded the best classification performance at a given array size for one particular task, was invariably outperformed by a different subset of detectors, and by the entire array, when used in at least one other vapor discrimination task. Arrays of detectors were nevertheless identified that yielded robust discrimination performance between compositionally close mixtures of 1-propanol and 2-propanol, n-hexane and n-heptane, and meta-xylene and para-xylene, attesting to the excellent analyte classification performance that can be obtained through the use of such semi-selective vapor detector arrays.
\end{abstract}

Keywords: vapor detectors, detector array size

\section{INTRODUCTION}

A significant issue in the use of arrays of semi-selective vapor detectors ${ }^{1,2}$ is the dependence of analyte classification performance on the number of detectors in the array. Patel et al. have claimed that four conducting polymer composite detectors are sufficient to provide a "universal solvent detector." 3 Park et al. found that for certain analyte classification tasks, various arrays of 3-6 surface acoustic wave detectors provided mutually comparable results, leading these authors to conclude that increasing the number of detectors in an array did not significantly improve classification performance. ${ }^{4}$ However, measurements using conducting polymer composite vapor detectors have indicated that the performance in certain vapor classification tasks can improve as the number of different detectors in the array is increased. ${ }^{5}$ The relationship between the number of detectors and overall system performance is important because significant engineering tradeoffs are faced for surface acoustic wave (SAW) devices, ${ }^{6-10}$ quartz crystal microbalances, ${ }^{9,11-12}$ conducting polymer detectors, ${ }^{13-14}$ and dye-impregnated optical beads ${ }^{15-16}$ or optical fibers ${ }^{17-18}$ as the number of detectors is increased. The focus of the work reported herein is to address in a quantitative fashion the performance of differently sized arrays of semi-selective vapor detectors for selected vapor detection tasks.

The vapor detectors that we have used for this purpose are chemically sensitive resistors fabricated from composites of conductors and insulating organic polymers. ${ }^{3,19-20}$ Sorption of an analyte into these materials produces a swelling of the film that affects the properties of the percolative network of conductive particles in the composite. The swelling produces a change in the dc electrical resistance of the detector that is readily read with a multiplexing dc ohmmeter. ${ }^{19}$ For data produced by an array of $d$ detectors, with one descriptor per detector (in our case the steady-state relative differential response value of the composite), the response, $X$, to each analyte presentation can be described as a $d$-dimensional vector:

$$
\mathbf{X}=\sum_{i=1}^{d} c_{i} x_{i}
$$

with the coefficient of the $i^{\text {th }}$ dimension of $\mathbf{X}, c_{i}$, equaling the response of the $i^{\text {th }}$ detector in the array.

To evaluate the magnitude of this metric, the points in a $d$-dimensional space are projected orthogonally onto a line, reducing the classification problem from $d$ dimensions to one dimension. When the data are projected onto one dimension, it is desirable to maximize the distance between the means of the two classes being separated, while minimizing their withinclass variation. Such a ratio can be expressed as a resolution factor, $R F$ (eq 2), where $\delta$ is the distance between the two class means, and $\sigma_{1}$ and $\sigma_{2}$ are the standard deviations of the two classes, respectively: ${ }^{21}$ Fisher's linear discriminant finds the 
vector $\mathbf{w}$ onto which the data are projected that maximizes the $R F$. This $R F$ value is an inherent property of the data and is independent of the algorithm that might be used subsequently to assign any individual data point to a class. ${ }^{21}$

$$
\mathrm{RF}=\frac{\delta}{\sqrt{\sigma_{1}^{2}+\sigma_{2}^{2}}}
$$

To compare quantitatively the relative performance of various detector arrays, the collection of arrays must be presented with pairs of analytes that will not be perfectly classified by at least some of the arrays. This was not the case with pairs of single-component organic vapors presented at relatively high concentration, all of which were perfectly (or nearly so) separated from each other, including structural isomers such as ortho- and meta-xylene. ${ }^{3,5,20,23}$ As part of this work, we have challenged a carbon black polymer composite detector array with a pair of compounds that are very chemically similar, $\mathrm{H}_{2} \mathrm{O}$ and $\mathrm{D}_{2} \mathrm{O}$. In addition, it is useful to consider the classification performance between mixtures of analytes. Several different binary mixtures of 1-propanol and 2-propanol, of n-hexane and n-heptane, and of meta-xylene and para-xylene were therefore utilized as part of the present work. Another method to decrease the discriminating ability of a detector array is to decrease the signal-to-noise ratio of the individual detectors.

\section{EXPERIMENTAL}

The acquisition and initial treatment of some of the data analyzed in this paper have been described in a prior article. ${ }^{22}$ One detector set used in this collection of experiments has been described previously and is designated herein as detector Set A (Table 1). ${ }^{22}$ A first data set collected with these detectors consisted of exposures to analytes each of which was either low in concentration or extremely similar to at least one other analyte in the study (Tasks 1-21, Table 2) Each analyte was exposed to the detectors 140 times for $300 \mathrm{~s}$ per exposure, with a separation of $600 \mathrm{~s}$ between exposures. The background gas was oil-free laboratory air that contained $1.10 \pm 0.15$ parts per thousand (ppth) of water vapor.

Table 1. Polymeric Component of Carbon Black Composite Vapor Detectors

\section{Set $\mathbf{A}$}

1. poly(ethylene-co-vinyl acetate), $70 \%$ vinyl acetate

2. poly(ethylene oxide)

3. poly(vinyl pyrrolidone), ${ }^{\text {a }}$ ABA block copolymer

4. 1,2-polybutadiene

5. polycaprolactone

6. poly(4-vinyl phenol) ${ }^{\mathrm{a}}$

7. poly(vinyl acetate)

8. cellulose acetate

9. poly(4-vinyl pyridine) ${ }^{\mathrm{a}}$

10. poly(methyl methacrylate ${ }^{\mathrm{a}}$

11. poly(styrene-co-maleic anhydride)

12. poly(vinyl butyral)

13. hydroxypropyl cellulose

14. ethyl cellulose

15. poly(ethylene-co-acrylid acid), $86 \%$ ethylene

16. poly(methyloctadecylsiloxane)

17. poly(ethylene glycol)

18. poly(ethylene-co-vinyl acetate) $18 \%$ vinyl acetate

19. polystyrene ${ }^{\mathrm{a}}$

20. poly(styrene-co-acrylonitrile) ${ }^{\mathrm{a}}$

\section{Set B}

1. polycaprolactone

2. poly(ethylene-co-vinyl acetate), $40 \%$ vinyl acetate

3. poly(ethylene oxide)

4. poly(ethylene glycol)

5. poly(styrene-co-butadiene), ABA block

copolymer, $30 \%$ styrene

6. poly(methyloctadecylsiloxane)

7. poly(vinyl stearate)

8. ethyl cellulose

9. Kraton $\mathrm{D}-1102^{\mathrm{b}}$

10. Kraton G-1652M

11. poly(4-vinyl phenol) ${ }^{c}$

12. poly(vinyl acetate)

13. poly(vinyl pyrrolidone) ${ }^{\mathrm{c}}$

14. polycarbonate

15. polystyrene ${ }_{c}$

16. polysulfone

17. poly(methyl methacrylate) ${ }^{c}$

18. poly(vinyl butyral) ${ }^{\mathrm{c}} \mathrm{d}^{\mathrm{d}}$

19. hydroxypropyl cellulose

20. poly(styrene-co-isoprene), $14 \%$ styrene

\footnotetext{
a Plasticized with $8 \%$ by mass bis(2-ethylhexyl) pthalate

${ }^{\mathrm{b}}$ Commercial polymers purchased from Shell Corp.

${ }^{\mathrm{c}}$ Plasticized with $50 \%$ by mass bis(2-ethylhexyl) pthalate

${ }^{\mathrm{d}}$ Plasticized with $50 \%$ by mass di(ethylene glycol) dibenzoate
} 
Table 2. Description of Tasks In Series I and Series II Data Sets

\begin{tabular}{|c|c|c|c|c|c|c|c|c|}
\hline \multirow[b]{2}{*}{ Task } & \multicolumn{4}{|c|}{ Analyte 1 } & \multicolumn{4}{|c|}{ Analyte 2} \\
\hline & Component 1 & $\overline{\mathrm{P} / \mathrm{P}^{\mathrm{o}}}$ & Component 2 & $\mathrm{P} / \mathrm{P}^{\mathrm{o}}$ & Component & $\overline{\mathrm{P} / \mathrm{P}^{\mathrm{o}}}$ & Component 2 & $\mathrm{P} / \mathrm{P}^{\mathrm{o}}$ \\
\hline$\overline{1}$ & $\mathrm{H}_{2} \mathrm{O}$ & $\overline{0.050}$ & $\mathrm{D}_{2} \mathrm{O}$ & 0.050 & & & & \\
\hline 2 & 1-propanol & 0.010 & 2-propanol & 0.010 & & & & \\
\hline 3 & 1-propanol & 0.0075 & 2-propanol & 0.0075 & & & & \\
\hline 4 & 1-propanol & 0.0050 & 2-propanol & 0.0050 & & & & \\
\hline 5 & 1-propanol & 0.0025 & 2-propanol & 0.0025 & & & & \\
\hline 6 & n-heptane & 0.010 & n-hexane & 0.010 & & & & \\
\hline 7 & n-heptane & 0.0075 & n-hexane & 0.0075 & & & & \\
\hline 8 & n-heptane & 0.0050 & n-hexane & 0.0050 & & & & \\
\hline 9 & n-heptane & 0.0025 & n-hexane & 0.0025 & & & & \\
\hline 10 & 1-propanol & 0.0025 & 2-propanol & 0.0025 & 1-propanol & 0.0023 & 2-propanol & 0.0027 \\
\hline 11 & 1-propanol & 0.0025 & 2-propanol & 0.0025 & 1-propanol & 0.0029 & 2-propanol & 0.0021 \\
\hline 12 & 1-propanol & 0.0025 & 2-propanol & 0.0025 & 1-propanol & 0.0015 & 2-propanol & 0.0035 \\
\hline 13 & 1-propanol & 0.0023 & 2-propanol & 0.0027 & 1-propanol & 0.0029 & 2-propanol & 0.0021 \\
\hline 14 & 1-propanol & 0.0023 & 2-propanol & 0.0027 & 1-propanol & 0.0015 & 2-propanol & 0.0035 \\
\hline 15 & 1-propanol & 0.0029 & 2-propanol & 0.0021 & 1-propanol & 0.0015 & 2-propanol & 0.0035 \\
\hline 16 & n-heptane & 0.0025 & n-hexane & 0.0025 & n-heptane & 0.0023 & n-hexane & 0.0027 \\
\hline 17 & n-heptane & 0.0025 & n-hexane & 0.0025 & n-heptane & 0.0029 & n-hexane & 0.0021 \\
\hline 18 & n-heptane & 0.0025 & n-hexane & 0.0025 & n-heptane & 0.0015 & n-hexane & 0.0035 \\
\hline 19 & n-heptane & 0.0023 & n-hexane & 0.0027 & n-heptane & 0.0029 & n-hexane & 0.0021 \\
\hline 20 & n-heptane & 0.0023 & n-hexane & 0.0027 & n-heptane & 0.0015 & n-hexane & 0.0035 \\
\hline 21 & n-heptane & 0.0029 & n-hexane & 0.0021 & n-heptane & 0.0015 & n-hexane & 0.0035 \\
\hline $22-23$ & 1-propanol & 0.0011 & 2-propanol & 0.00090 & 1-propanol & 0.00090 & 2-propanol & 0.0011 \\
\hline 24-25 & n-heptane & 0.0011 & n-hexane & 0.00090 & n-heptane & 0.00090 & n-hexane & 0.0011 \\
\hline 26-27 & m-xylene & 0.0011 & p-xylene & 0.00090 & m-xylene & 0.00090 & p-xylene & 0.0011 \\
\hline 28 & 1-propanol & 0.0075 & & & 2-propanol & 0.0083 & & \\
\hline 29 & 1-propanol & 0.075 & & & 2-propanol & 0.083 & & \\
\hline
\end{tabular}

${ }^{a}$ Concentrations are listed in parts per million in air, as determined from $\mathrm{P} / \mathrm{P} \circ$ values. $\mathrm{P} \circ$ values were calculated at a temperature of $21^{\circ} \mathrm{C}$ and a pressure of 750 torr.

The initial exposures of each run tended to give responses that varied more than those later in the run. For this reason, the first 40 exposures to each analyte in this data set were rejected. The remaining 100 exposures to each analyte, which were mutually similar within a run, were used for analysis of this data set. Each task was run separately, resulting in all of the exposures from task 1 being run before any of the exposures of task 2, for example. Within a single task, the analytes were presented in random order over the 200 exposures (100 to each analyte) of the task. Table 2 presents a summary of the 21 tasks (1-21) that are collectively denoted as the Series I data runs.

In a separate set of experiments, binary mixtures of n-hexane/n-heptane, 1-propanol/2-propanol, and meta-xylene/para-xylene were exposed to a different array of conducting polymer composite detectors. The detector array used in these runs, denoted as detector Set B, consisted of 80 detectors that were housed in two separate chambers. The 80 detectors were formed from 4 nominally identical copies of each of 20 compositionally distinct detector materials (Table 1). Detectors were fabricated using procedures described previously. ${ }^{5,19,23,24}$ Except where otherwise noted, only data from the first set of 20 compositionally different detectors were analyzed as detector Set B data.

In these runs, designated collectively as the Series II data runs, each analyte was exposed 200 times to the detector array, with each individual exposure consisting of $70 \mathrm{~s}$ of exposure to laboratory air, $80 \mathrm{~s}$ of exposure to the analyte of interest, and then $60 \mathrm{~s}$ of exposure to laboratory air. In the last two runs of the Series II data (tasks 28-31 in Table 2), 1-propanol and 2-propanol were exposed separately to the detectors at relatively high and relatively low vapor phase concentrations that are listed in Table 2 . In these runs, each analyte was exposed 200 times to a single set of 20 detectors, with each exposure consisting of $40 \mathrm{~s}$ of flowing background laboratory air, $70 \mathrm{~s}$ of analyte, followed by $40 \mathrm{~s}$ of laboratory air. 
All data were baseline corrected to eliminate drift effects within single analyte exposures. For each exposure in the Series I data set, the baseline resistance, $R_{b}$, of each detector was calculated from the average of the resistance readings for the $60 \mathrm{~s}$ immediately prior to the beginning of the analyte exposure. The equilibrium response, $R_{\text {eq }}$, was calculated from the average of the resistance readings for the last $60 \mathrm{~s}$ of the exposure to analyte vapor. For runs in the Series II data set, which used shorter exposure times, $R_{b}$ was determined from the average of the resistance readings in the last $30 \mathrm{~s}$ immediately prior to each exposure, and $R_{\mathrm{eq}}$ was determined from data obtained in the last $30 \mathrm{~s}$ of each analyte vapor exposure. Baseline correction of Series II data was performed by fitting a regression line to the pre-exposure resistance readings, and correcting all subsequent data points by the difference in the value of the regression fit at the time of the measurement of that data point and at $t=0$.

The quantity used in analysis of both Series I and Series II data was the steady-state relative differential resistance change, $\Delta R / R_{b}$, where $\Delta R=R_{e q}-R_{b}$. The signal-to-noise $(S / N)$ ratio for each detector at the analyte concentration of interest was computed by determining the ratio between the $\Delta R$ value determined from a single exposure to the analyte of interest and 3 times the standard deviation of the corrected baseline resistance. The classification performance, $\mathcal{P}$, of the detector arrays was evaluated using different methods for the different data series. Because only 100 exposures to each analyte were available in the Series I data set, the array classification performance from these data was evaluated using the leave-one-out (LOO) cross validation procedure. This process provides an approach in which both training set data and test set data can be obtained from a data set in which the classes of all members are known. ${ }^{21}$ In the LOO approach, one member is removed from a data set of $n$ members, the classifier is developed using the $n-1$ remaining members of the data set, and the resulting classifier is used to assign the withheld member to a class. A second member of the data set is then withheld, a new classifier is constructed from the $n-1$ remaining members, and this classifier is then used to assign that member to a class. The procedure is repeated through all $n$ members of the data set. The fraction of correct assignments out of the $n$ possible cases provides an evaluation of the LOO classification performance $\mathscr{R}$ of a given array towards a specific pair of analytes utilizing a given discriminant algorithm.

For the Series II data, 200 exposures were available for each analyte. This larger data set facilitated reliable use of independently constructed test and training sets to evaluate the classification performance of the various arrays of interest. 100 of the 200 total exposures to each analyte were randomly selected to form the training set for that analyte. The remaining exposures formed the test set for that particular analyte. The training set was used to formulate a Fisher linear discriminant decision boundary (a hyperplane orthogonal to the Fisher discriminant vector) which was then evaluated with respect to analyte classification performance on the exposures in the test set. The classification performance on the training set is denoted as $\mathscr{P}_{\text {trn }}$ and that on the test set is denoted as $\mathscr{P}_{\text {tst }}$. The capability of the model with respect to a given training set was determined by the Fisher $R F$ value; the capability with regard to a test set was determined by the fraction of the exposures that were correctly classified using the decision boundary developed on the training set. The Series II data were also analyzed separately using the leave-one-out procedure to facilitate direct comparison with the classification performance resulting from analysis of the Series I data.

\section{RESULTS}

3.1 Classification of $n$-Hexane/n-Heptane and 1-Propanol/2-Propanol Mixtures as a Function of the Number of Different Detectors in the Array

Figure 1 displays the classification performance for Detector Set A discriminating a mixture of $\mathrm{n}$ hexane at $\mathrm{P} / \mathrm{P}^{\circ}=0.025$ and $\mathrm{n}$-heptane at $\mathrm{P} / \mathrm{P}^{\circ}=0.025$ from a mixture of $\mathrm{n}$-hexane at $\mathrm{P} / \mathrm{P}^{\circ}=0.027$ and $\mathrm{n}$-heptane at $\mathrm{P} / \mathrm{P}^{\circ}=0.023$ (task 16, Table 2). The classification performance for this task was calculated using the leave-oneout cross validation procedure on the entire set of 100 Series I exposures to each analyte mixture. The full 20 detector Set A array yielded 78 percent correct classification for the 200 exposures of this discrimination task. This value is denoted as $\mathscr{P}_{1}\{[\ddot{E}(20)] \Rightarrow 16\}$, where the argument of $\ddot{E}$ indicates the number of unique detectors in the array, $\ddot{E}$, and the $\Rightarrow \mathbf{1 6}$ notation indicates that the leave-one-out classification performance, $\mathscr{R}$, of this array was evaluated for classification task 16 in Table 2 . For each value of $k$ in the range $1 \beta k \beta 20$, where $k$ is the number of compositionally different detectors in the array, an exhaustive search of all possible $((20 !) /[(k !)(20-k !)]) k$-detector combinations from the 20-detector Set A array was performed to identify the array having $\mathrm{k}$ detectors that had the best leave-one-out classification performance for the task of concern. This array, denoted $\left[\ddot{E}_{\max }{ }^{16}(\mathrm{k})\right]$ for each value of $\mathrm{k}$, had a classification performance $\mathscr{R}\left\{\left[\ddot{E}_{\max }{ }^{16}(\mathrm{k})\right] \Rightarrow 16\right\}=\mathscr{R}_{\text {, } \max }{ }^{16}$ (k). Figure 1 depicts the relative classification performance, $\mathscr{P}_{\mathrm{rel}}{ }^{16}(\mathrm{k})$, of these optimally performing array subsets of Detector Set A as a function of array size, with the relative classification performance of each 
selected subset representing the classification performance of $\left[\mathrm{E}_{\max }{ }^{16}(\mathrm{k})\right]$ normalized relative to that of the full 20 detector array (eqn 3 ):

$$
\mathcal{P}_{\text {rel }}{ }^{16}(\mathrm{k})=\left(\mathscr{R}_{1}\left\{\left[\ddot{\mathrm{E}}{ }_{\text {max }}{ }^{16}(\mathrm{k})\right] \Rightarrow 16\right\} /\{\mathscr{R}\{[\ddot{\mathrm{E}}(20)] \Rightarrow 16\})=\mathscr{R}_{1, \max }{ }^{16}(\mathrm{k}) /(\{\mathscr{R}\{[\mathrm{E}(20)] \Rightarrow 16\})\right.
$$

Table 3 lists the best-performing detector sets for each value of $\mathrm{k}$ in the range $1 \beta \mathrm{k} \beta 10$ as well as the leave-oneout classification performance of these detector sets for this particular analyte separation task. Figure 1 clearly indicates that for these detector arrays, the classification performance for an individual task either increased or did not decrease significantly as the number of chemically different detectors increased.

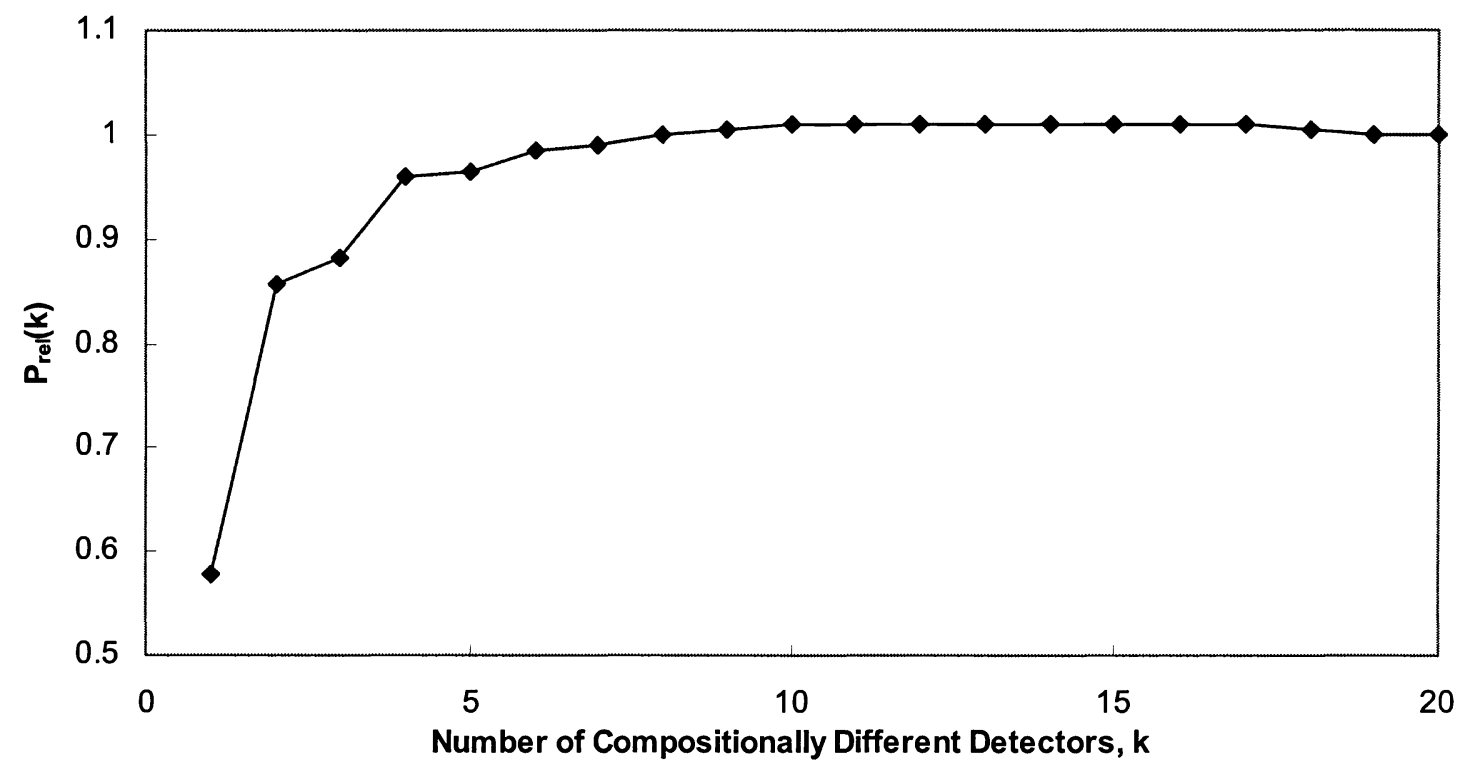

Figure 1. Classification performance vs. array size for distinguishing a mixture of $\mathrm{n}$-hexane at $\mathrm{P} / \mathrm{P}^{\circ}=0.025$ and $\mathrm{n}$-heptane at $\mathrm{P} / \mathrm{P}^{\circ}=0.025$ from a mixture of $\mathrm{n}$-hexane at $\mathrm{P} / \mathrm{P}^{\circ}=0.027$ and $\mathrm{n}$ heptane at $\mathrm{P} / \mathrm{P}^{\circ}=0.023$. The classification performance for this task of the best-performing $\mathrm{k}$ member detector set is plotted relative to that of the full 20-detector array.

Table 3a. Detectors That Provided Maximum Classification Performance at Various Array Sizes For Task 16

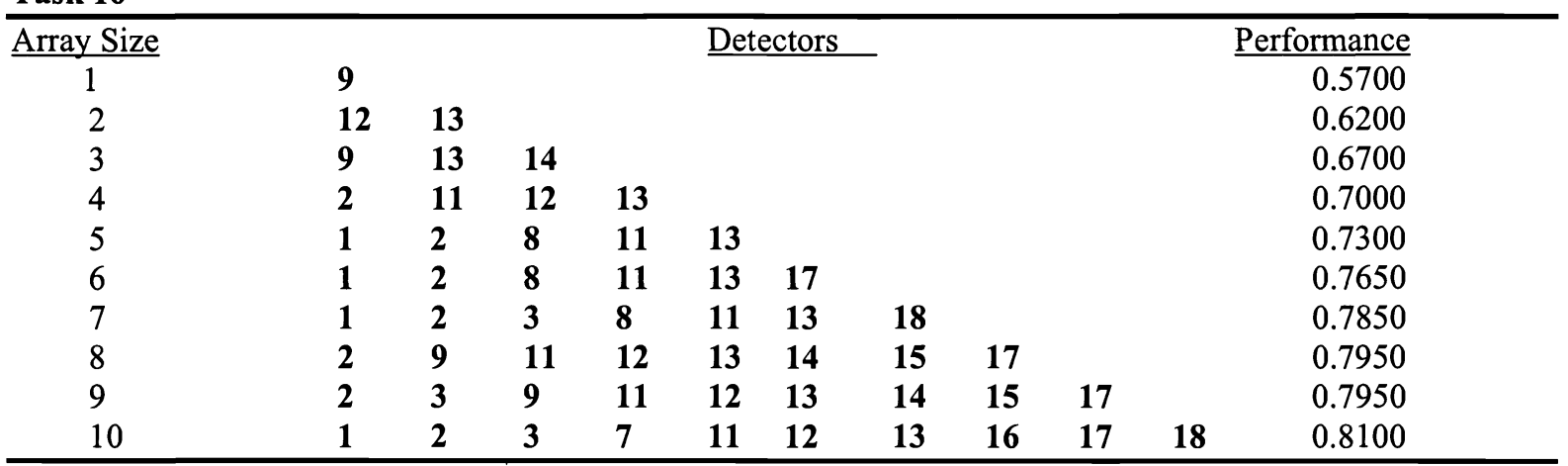

Figure 2 displays analogous data for Detector Set $\mathrm{B}$, in which a mixture of $\mathrm{n}$-hexane at $\mathrm{P} / \mathrm{P}^{\circ}=0.011$ and $n$-heptane at $\mathrm{P} / \mathrm{P}^{\circ}=0.0090$ is separated from a mixture of $\mathrm{n}$-hexane at $\mathrm{P} / \mathrm{P}^{\circ}=0.0090$ and $\mathrm{n}$-heptane at $\mathrm{P} / \mathrm{P}^{\circ}=0.011$ (Task 24, Table 2). The classification performance $P_{1}\{[\ddot{E}(20)] \Rightarrow \mathbf{2 4}\}$ was evaluated for the full 20 detector Set B using the leave-one-out procedure (to aid in comparison with Data Set I results), and this array yielded 95 percent correct classification for this task. An exhaustive search was then performed to identify the subset of detectors of a given array size, designated as $\left[\ddot{E}_{1, \max }{ }^{24}(\mathrm{k})\right]$, that yielded the best leave-one-out cross-validation 
performance for each value of $k$. The classification performance, $\mathscr{A}_{\text {, } \max }{ }^{24}(\mathrm{k})$, of $\left[\ddot{E}_{1, \max }{ }^{24}(\mathrm{k})\right] \Rightarrow 24$, normalized relative to the performance of the full 20 -detector array for this task is plotted for $1 \beta \mathrm{k} \beta 20$. Table $3 \mathrm{~b}$ lists the best-performing detector sets for each value of $\mathrm{k}$ in the range $1 \beta \mathrm{k} \beta 10$ as well as the leave-one-out classification performance of these detector sets for this particular analyte separation task. Similar trends were observed for essentially all of the other tasks evaluated in this work, with the exception of the xylenes separation tasks (tasks 25 and 26 in Table 2) of Data Set II.

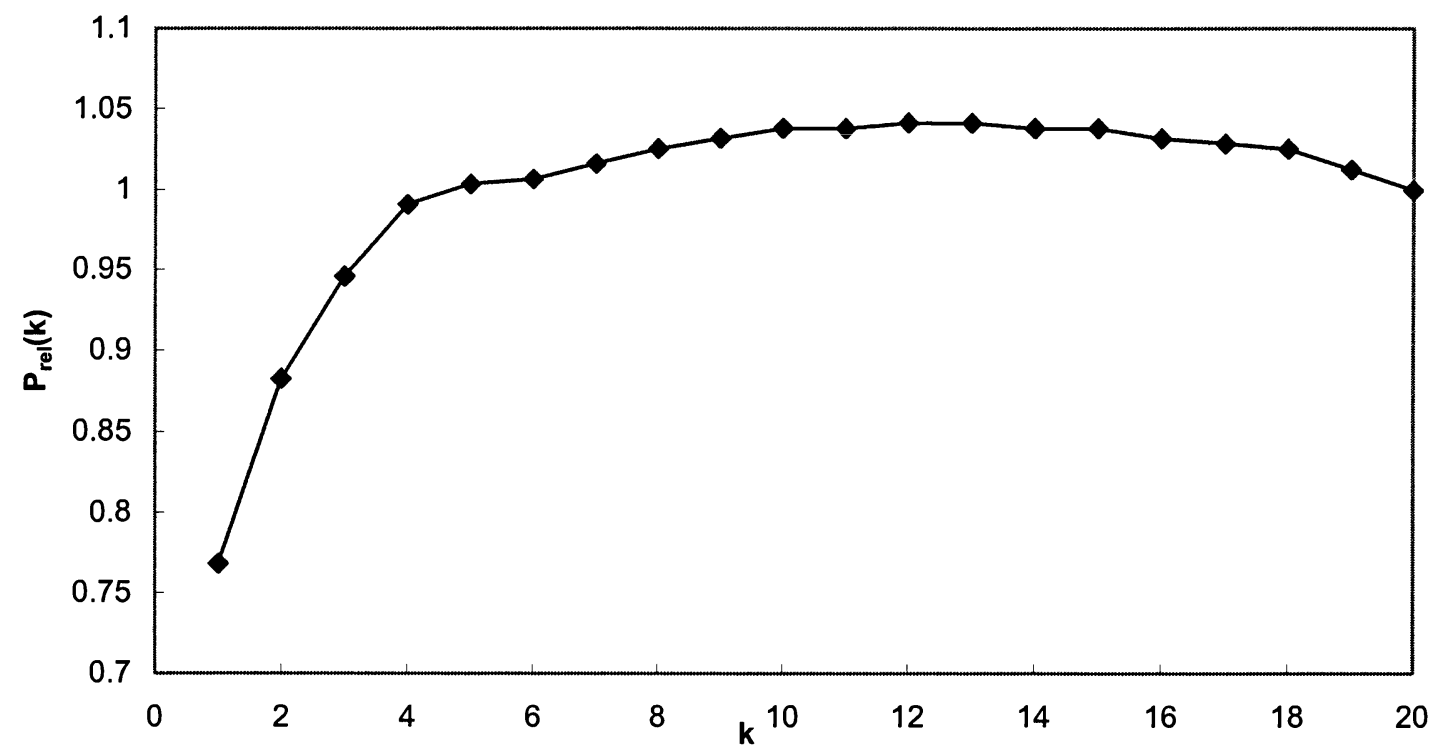

Figure 2. Classification performance vs. array size for distinguishing a mixture of $n$-hexane at $\mathrm{P} / \mathrm{P}^{\circ}=0.025$ and $\mathrm{n}$-heptane at $\mathrm{P} / \mathrm{P}^{\circ}=0.025$ from a mixture of $\mathrm{n}$-hexane at $\mathrm{P} / \mathrm{P}^{\circ}=0.027$ and $\mathrm{n}$ heptane at $\mathrm{P} / \mathrm{P}^{\circ}=0.023$. The classification performance for this task of the best-performing $\mathrm{k}$ member detector set is plotted relative to that of the full 20 -detector array.

Table 3b. Detectors That Provided Maximum Classification Performance at Various Array Sizes For Task 24

\begin{tabular}{|c|c|c|c|c|c|c|c|c|c|c|c|}
\hline Array Size & & & \multicolumn{6}{|c|}{ Detectors } & \multirow{2}{*}{\multicolumn{3}{|c|}{$\frac{\text { Performance }}{06375}$}} \\
\hline 1 & 7 & & & & & & & & & & \\
\hline 2 & 19 & 20 & & & & & & & & & 0.8750 \\
\hline 3 & 8 & 19 & 20 & & & & & & & & 0.9175 \\
\hline 4 & 8 & 14 & 19 & 20 & & & & & & & 0.9225 \\
\hline 5 & 8 & 11 & 15 & 19 & 20 & & & & & & 0.9350 \\
\hline 6 & 1 & 7 & 8 & 15 & 19 & 20 & & & & & 0.9375 \\
\hline 7 & 1 & 2 & 7 & 8 & 15 & 19 & 20 & & & & 0.9400 \\
\hline 8 & 1 & 2 & 6 & 7 & 8 & 15 & 19 & 20 & & & 0.9450 \\
\hline 9 & 1 & 2 & 3 & 6 & 7 & 8 & 15 & 19 & 20 & & 0.9475 \\
\hline 10 & 1 & 3 & 7 & 8 & 9 & 11 & 12 & 15 & 19 & 20 & 0.9500 \\
\hline
\end{tabular}

\subsection{Relative Classification Performance of "Optimal" Five-Detector Arrays in Vapor Discrimination Tasks}

Figures 1-2 also indicate that nearly optimal classification performance could be obtained in many cases by selecting subsets of 4-10 detectors, and then only using response data from that subset of detectors to classify presentations of that particular pair of analytes to the detector array. However, as shown in Tables 3a$\mathrm{b}$, the collection of detectors in the array of a given size that produced the best classification performance for the hexane/heptane mixture classification task was not the same as that optimized for the 1-propanol/2-propanol mixture classification task. To assess the generality of this observation, an extensive comparison was performed of the classification performance of selected detector array subsets in a diverse collection of different analyte classification tasks. 
For specificity, this analysis concentrated on evaluating the performance of arrays that consisted of 5 compositionally different detectors obtained from the full collection of $20 \mathrm{Set}$ B detectors. The performance of each of $(20 !) /[(15 !)(5 !)]$ such 5-detector arrays was evaluated for the first six separate classification tasks in the Series II data set (Tasks 22-27, Table 2). The detector subset chosen was that which maximized the Fisher $R F$ value resulting from the training data set for the task of interest. These 5-detector arrays are designated as $\left[\ddot{E}_{t r n, m a x}{ }^{J}(5)\right]$, where the subscript $t r n$ indicates that the arrays were selected based on their abilities to maximize the $R F$ values of the training set data, and the bold superscript numeral $\mathbf{J}$ designates the task for which the array was identified as providing the maximum Fisher $R F$ value.

The classification performance of each $\left[\ddot{E}_{t r n, m a x}(5)\right]$ array was then evaluated on an independent test set of exposures that included 6 analyte classification tasks (22-27) of the Series II data. For each combination of a 5-detector array $\left[\ddot{E}_{t r n}{ }_{\text {max }} \mathbf{J}(5)\right]$ and an individual pairwise separation task $\mathbf{K}$ of the Series II data, a new Fisher model was determined based on the 200 analyte exposures of the training set for task K presented to the detector set $\left[\ddot{E}_{\text {trn, max }}{ }^{\mathbf{J}}(5)\right]$. The classification performance $\mathscr{P}_{\text {st }}\left\{\left[\ddot{E}\right.\right.$ trn,max $\left.\left.{ }^{\mathbf{J}}(5)\right] \Rightarrow \mathbf{K}\right\}$ was then evaluated by determining the fraction of a separate 200 exposure task $\mathbf{K}$ test data set that were on the correct side of the Fisher discriminant boundary. The process was repeated for all $J$ and $K$ in the range $22{ }_{\beta} J_{\beta} 27$ and $22 \beta K K_{\beta} 27$.

The resulting detector performance data are summarized in the two $6 \times 6$ matrices of Table 4.

The entries in each column designate the performance, $\mathscr{P}_{\mathrm{tst}}\left\{\left[\ddot{\mathrm{E}}_{\mathrm{trn}, \max }{ }^{\mathbf{J}}(5)\right] \Rightarrow \mathbf{K}\right\}$, of each $\left[\ddot{E}_{\mathrm{tm}, \max }{ }^{\mathbf{J}}(5)\right]$ array when used in each of the six tasks $\left(22 \beta K_{\beta} 27\right)$ evaluated in the Series II data run. Two matrices are presented, one of

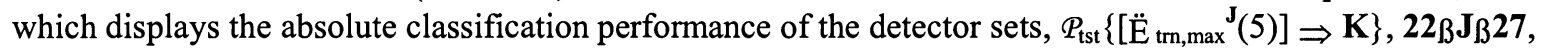
$22 \beta K_{\beta} 27$, and the other of which displays the classification performance of the detector sets for each task $K$ when normalized to the value of $\mathscr{P}_{\text {st }}\left\{\left[\ddot{E}_{\text {trn,max }} \mathbf{J}(5)\right] \Rightarrow \mathbf{K}\right\}$ obtained when $\mathbf{K}=\mathbf{J}$. In other words, for each test set task $\mathbf{K}$, the test set classification performance of the various 5-detector arrays $\left[\ddot{E}_{\text {trn,max }}{ }^{\mathbf{J}}(5)\right]$ was normalized relative to the test set classification performance of the array, [ $\left.\ddot{E}_{t r n, \max }{ }^{J}(5)\right]$, that yielded the maximum training set Fisher RF value for that particular analyte classification task. The normalization accounts for inherent differences in the difficulties of the various classification tasks being evaluated. Furthermore, because the task is a two-class classification problem, a classification performance value of 0.5 is equal to that of random chance, so normalization was performed using the formula:

$$
\left(\mathscr{P}_{\text {tst }}\left\{\left[\ddot{E}_{\text {trn,max }}{ }^{\mathrm{J}}(5)\right] \Rightarrow \mathbf{K}\right\}-0.5\right) /\left(\mathscr{P}_{\text {tst }}\left\{\left[\ddot{E}_{\text {trn,max }} \mathbf{J}(5)\right] \Rightarrow \mathbf{K} \text { for } \mathbf{K}=\mathbf{J}\right\}-0.5\right)
$$

The entries along the diagonal of the normalized matrix are all 1.0, because by definition they are the values to which the test set classification performance for a given task have been normalized.

The data indicate that different detector subsets yielded the best classification performance for different tasks. An equivalent statement is that the combination of five detectors which produced the best classification performance for one task was significantly outperformed by another 5-detector set in another, different task. For example, the combination of 5 detectors, $\left[\ddot{E}_{t n, m a x}{ }^{27}(5)\right]$, that produced the best Fisher RF value in a training set run of the pair of $m$-xylene/ $p$-xylene mixtures yielded only $34-38 \%$ of the normalized classification performance on 1-propanol/2-propanol test set mixtures (tasks 22, 23) relative to the 1propanol/2-propanol mixture test set classification performance (tasks 22, 23) of the 5-detector combination, [ $t_{t r n, \max }^{22}(5)$ or [ $\left.\left.\ddot{E}_{t r n, m a x}{ }^{23}(5)\right]\right]$, that produced the best training set Fisher RF value for each 1-propanol/2-propanol mixture discrimination task (Table 4). Similarly, the 5-detector set that yielded the best Fisher RF value for the other training set of $m$-xylene/ $p$-xylene exposures, [ $\left.\ddot{E}_{t n, \max }{ }^{26}(5)\right]$, only yielded $22-40 \%$ of the classification performance on 1-propanol/2-propanol test set mixtures (tasks 22, 23) relative to the 1-propanol/2-propanol mixture test set performance of the 5-detector set $\left(\left[\ddot{E}_{t n, \text { max }}{ }^{22}(5)\right]\right.$ or $\left.\left[\ddot{E}_{t r n, m a x}{ }^{23}(5)\right]\right)$ that yielded the best Fisher $\mathrm{RF}$ value on the training sets of these 1-propanol/2-propanol mixture classification tasks. Similarly, the 5detector sets that were found to yield the best Fisher RF value for either of the $n$-hexane/n-heptane training set mixture tasks, $\left[\ddot{E}_{t r n, m a x}{ }^{24}(5)\right]$ and $\left[\ddot{E}_{t r n, m a x}{ }^{25}(5)\right]$, yielded much worse test set classification performance in the 1propanol/2-propanol classification tasks $(22, \mathbf{2 3})$ than was produced when either of the 5-detector sets $\left(\left[\ddot{E}_{t r n, m a x}{ }^{22}(5)\right]\right.$ and $\left.\left[\ddot{E}_{t r, m a x}{ }^{23}(5)\right]\right)$ that produced the best Fisher RF value on the 1-propanol/2-propanol training set tasks $(\mathbf{2 2}, \mathbf{2 3})$ were used on the test set of 1-propanol/2-propanol mixture tasks $(\mathbf{2 2}, \mathbf{2 3})$.

\subsection{Classification Performance as a Function of the Number of Compositionally Different Detectors in the Array}

Given that different k-detector subsets were observed to produce the best classification performance for different tasks, and that classification performance in general either increased or did not decrease significantly 
as the number of chemically different detectors increased (Figures 1-2), it is of interest to compare the performance of subsets of detectors to the performance of a full array of 20 compositionally different detectors. This comparison was performed for all of the different tasks and data sets collected during the course of this work.

Table 4. Classification Performance of Arrays Formed from Five Compositionally Different Detectors Array

\begin{tabular}{|c|c|c|c|c|c|c|c|}
\hline & & 22 & 23 & 24 & 25 & 26 & 27 \\
\hline Designation & Detectors & \multicolumn{6}{|c|}{ Absolute Performance } \\
\hline$\left[\ddot{E}_{t r n m a x}^{22}(5)\right]$ & $8,9, \overline{11,18,19}$ & 0.735 & 0.755 & 0.755 & 0.815 & 0.850 & 0.880 \\
\hline$\left[\ddot{E}_{t r n, \max }^{23}(5)\right]$ & $8,11,13,18,19$ & 0.760 & 0.765 & 0.755 & 0.795 & 0.810 & 0.865 \\
\hline$\left[\ddot{\mathrm{E}}_{\mathrm{trn}, \max }{ }^{24}(5)\right]$ & $1,8,15,19,20$ & 0.620 & 0.560 & 0.930 & 0.930 & 0.760 & 0.850 \\
\hline$\left[\ddot{E}_{\mathrm{trn}, \max }{ }^{25}(5)\right]$ & $1,3,4,19,20$ & 0.670 & 0.565 & 0.890 & 0.925 & 0.655 & 0.695 \\
\hline$\left[\ddot{E}_{\mathrm{tr}, \max }^{26}(5)\right]$ & $4,8,9,12,18$ & 0.595 & 0.560 & 0.705 & 0.655 & 0.865 & 0.835 \\
\hline 5)] & $1,8,9,12,19$ & 0.590 & 0.600 & 0.705 & 0.740 & 0.815 & 0.880 \\
\hline
\end{tabular}

Designation

$\left[\ddot{E}_{\text {trn,max }}^{22}(5)\right]$

$\left[\ddot{E} \operatorname{trn}_{\max }^{23}(5)\right]$

$\left[\ddot{E ̈}_{\text {trn,max }} 24(5)\right]$

$[\ddot{E}$ trn,max $25(5)]$

$\left[\ddot{E} t_{\text {trm,max }}^{26}(5)\right]$

$\left[\ddot{E}_{t m, \text { max }}{ }^{27}(5)\right]$

${ }^{a}$ Due to differences in inherent task difficulty, prediction abilities for each task are

Normalized Performance $^{\mathrm{a}}$

$\begin{array}{llllll}\mathbf{1 . 0 0} & \mathbf{0 . 9 6 2} & 0.593 & 0.741 & 0.959 & 1.00 \\ \mathbf{1 . 1 1} & \mathbf{1 . 0 0} & 0.593 & 0.694 & 0.849 & 0.961 \\ 0.511 & 0.226 & \mathbf{1 . 0 0} & \mathbf{1 . 0 1} & 0.712 & 1.01 \\ 0.723 & 0.245 & \mathbf{0 . 9 0 7} & \mathbf{1 . 0 0} & 0.425 & 0.513 \\ 0.404 & 0.226 & 0.477 & 0.365 & \mathbf{1 . 0 0} & \mathbf{0 . 8 8 2} \\ 0.383 & 0.337 & 0.477 & 0.565 & \mathbf{0 . 8 6 3} & \mathbf{1 . 0 0}\end{array}$

normalized, with 1.00 representing a task being applied to its self-optimized set of 5 sensors.

Normalization was accomplished from the formula Value ${ }_{\text {norm }}=\left(\right.$ Value $\left._{\text {raw }}-0.5\right) /(\mathrm{Norm}-0.5)$, where "Norm" represents the number by which Value ${ }_{\text {raw }}$ is to be normalized.

First, the leave-one-out classification performance of the array of 20 chemically different detectors in detector set A was evaluated for each of the 21 tasks for which these detectors were used (Table 2, tasks 1-21). This returned a set of performance values $\mathscr{R}\{[\ddot{E}(20)] \Rightarrow \mathbf{J}\}$ for each task $\left(\mathbf{1}_{\beta} \mathbf{J}_{\boldsymbol{B}} \mathbf{2 1}\right)$. The leave-one-out classification performance for every individual combination of $k$-detectors, where $1 \beta \mathrm{k} \beta 20$ for each of the 21 tasks, was then also evaluated. For every task, the classification performance for each individual combination of k-detectors, $\mathscr{R}\left\{\left[\ddot{E}_{\mathrm{i}}(\mathrm{k})\right] \Rightarrow \mathbf{J}\right\}$ for $1 \beta$ i $\beta[(20 !) /(\mathrm{k} !)(20-\mathrm{k} !)]$, was then compared to that of the full 20 detector array, $\mathbb{R}\{[\ddot{E}(20)] \Rightarrow J\}$ for that same task. For each task, the classification performance for any k-detector array, $\mathscr{R}\left\{\left[\ddot{E}_{i}(\mathrm{k})\right] \Rightarrow \mathbf{J}\right\}$, was then normalized by dividing by the classification performance of the full 20 detector Set A array on that same task, $\mathscr{R}\{[\ddot{E}(20)] \Rightarrow \mathbf{J}\}$. These performance ratios were tabulated and used to create a function $\mathrm{g}(\mathrm{k})$ for which, by definition, no combination of $\mathrm{k}$ detectors does strictly better than $\mathrm{g}(\mathrm{k})$ relative to the full 20-detector Set A array on all 21 tasks of the Series I data run. Therefore, when an array containing $\mathrm{k}$ detectors is used to perform a set of tasks, at least one task among the set will yield a classification performance no better than $\mathrm{g}(\mathrm{k})$ relative to performance of the full 20 detector array, regardless of how the $k$ detectors are chosen. As displayed in Figure 3, no combination of $\mathrm{k}$ detectors with $1 \beta \mathrm{k}<20$ performed as well as the full 20-detector array on all of the tasks evaluated. Additionally, the value of $\mathrm{g}(\mathrm{k})$ more closely approached that of the full array as $k$ increased. Also, $g(k)>g(k-1)$ for $2 \leq k \leq 20$, hence increasing the size of the detector array always resulted in an increase in the value of $\mathrm{g}(\mathrm{k})$.

\subsection{Improvement in Classification Performance Upon Addition of Compositionally Different} Detectors to an Array Relative to Addition of Nominally Identical Copies of Detectors to an Array

Some of the improvement in classification performance displayed by the full 20 detector array relative to the performance of $k$-detector subsets $(k<20)$ for either the Set A or Set B detectors could possibly result from the larger number of observations that are used in the analysis process for a given measurement task when a constant number of data points is acquired from more total detectors. To this end, the six 5-detector sets $\left[\ddot{E}_{t r, m a x}(5)\right]$ for $22 \beta J_{\beta} 27$ (Table 4) that yielded the best Fisher discriminant $R F$ value for the training set data of each task for $22 \beta J_{\beta} 27$, respectively, were used to generate six 20 detector arrays, [Ë trm,max $\mathbf{J}(5 \times 4)$ ] for $22 \beta J_{\beta} 27$, by analyzing additionally the data produced by the three nominally identical copies of each detector 
composition that were available in the full 80-detector array of the Set B detectors. The weights for each detector in these 20 detector arrays were then independently determined using the training set data for each task of interest $\left(22 \beta J_{\beta} 27\right)$. The test set classification performance of these six 20 -detector $\left[\ddot{E}\right.$ trn,max $J^{J}(5 \times 4)$ ] arrays was then evaluated for each task in the range $22_{\beta} J_{\beta} 27$ of the Series II data, and the test set classification performance of these arrays was compared to the test set classification performance $\mathscr{P}_{\mathrm{tst}}\{[\ddot{\mathrm{E}}(20)] \Rightarrow \mathbf{J}\}$, $22 \beta J_{\beta} 27$, produced by the first set of 20 compositionally different detectors in the Set $B$ array. Table 5 presents the results of this comparison for the absolute and normalized classification performance of these 20detector arrays, with the normalization performed using the approach of eq (4) to account for inherent differences in difficulty between tasks as well as to account for the two-class character of the discrimination tasks being evaluated.

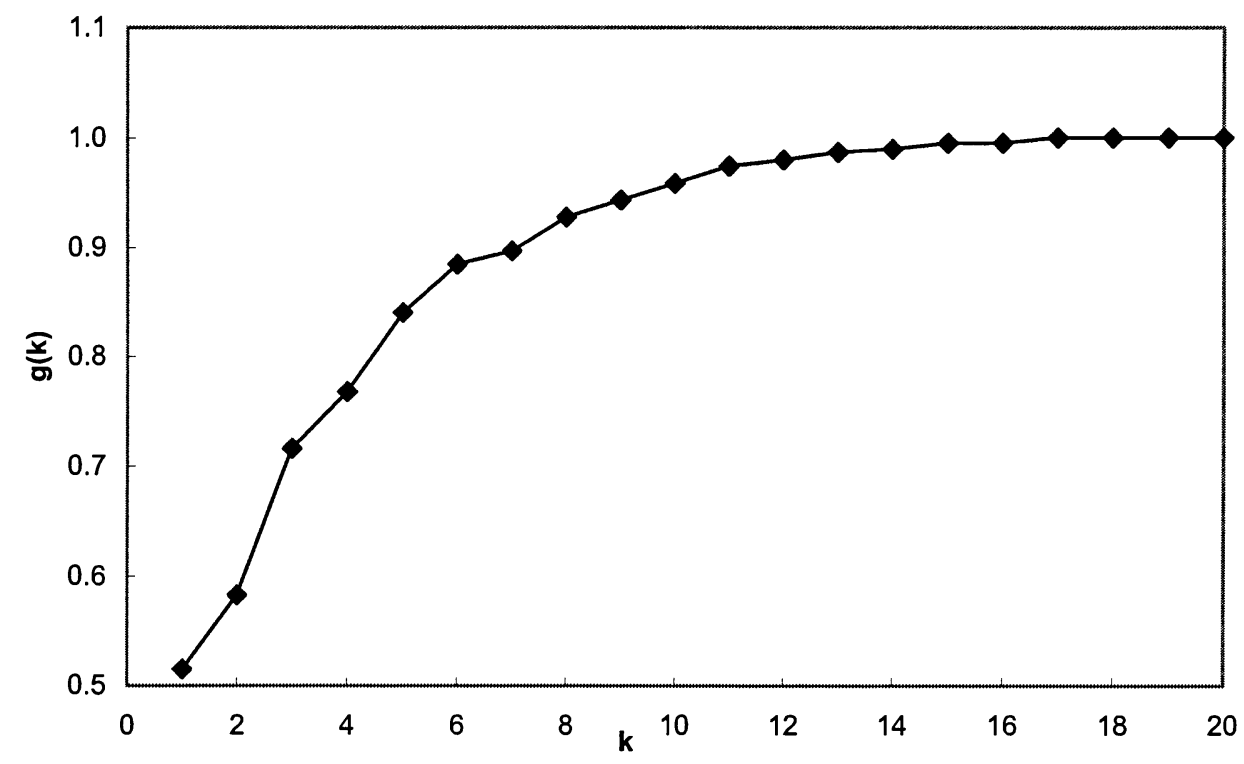

Figure 3. Worst normalized classification performance of any Set I task vs. array size $k$. For each task, normalized classification performance is relative to that of the full 20-detector Set A array. No combination of $k$ detectors does strictly better than $g(k)$ relative to the full 20 detector Set A array on all 21 tasks of the Series I data run.

Comparison of Tables 4 and 5 indicates that the mean absolute classification performance for a given task increased by $囚 1 \%$ when 3 additional copies of each detector were included in the array. Furthermore, the benefit of dimensionality reduction was clearly evident in that the $[\ddot{E}$ trn,max $\mathbf{J}(5 \times 4)] 20$-detector arrays always yielded better classification performance than $[\ddot{E}(20)]$ in the task $\mathbf{J}$ for which the $\left[\ddot{E}_{t r n}, \mathbf{m a x}^{\mathbf{J}}(5)\right]$ detector arrays were selected as providing the best test set classification performance for 5-detector arrays (Table 5).

Similarly, arrays that were identified as producing the optimal Fisher RF value on training set data for a specific task yielded excellent test set classification performance relative to $[\ddot{E}(20)]$ in a duplicate trial of that same task.

However, these 20 -detector [ $\left.\ddot{E}_{\mathrm{trn}, \max } \mathrm{J}(5 \times 4)\right]$ arrays generally yielded inferior test set classification performance relative to the set of 20 compositionally different Set B detectors when the specific 20-detector $\left[\ddot{E}_{t r n, m a x} \mathbf{J}(5 \times 4)\right]$ arrays were used for other tasks in the Series II data run. For example, the set of 20 compositionally different Set B detectors yielded $₫ 13 \%$ better test set classification performance than did any of the $\left[\ddot{E}_{t r n, m a x}{ }^{J}(5 \times 4)\right], 24 \beta J_{\beta} 27$ detector sets for either of the 1-propanol/2-propanol mixture classification tasks $(\mathbf{J}=\mathbf{2 2 , 2 3})$. Similarly, the detector sets $\left[\ddot{E}{ }_{t r n, m a x}{ }^{J}(5 \times 4)\right]$ that yielded optimal training set Fisher RF values for the heptane/hexane mixture classification tasks, $\mathbf{J}=\mathbf{2 4}, \mathbf{2 5}$, outperformed [ت̈ (20)] in test set classification performance $\mathscr{P}_{\text {tst }}\left\{\left[\ddot{E}_{\text {trn,max }} \mathbf{J}(5 \times 4)\right] \Rightarrow \mathbf{K}\right\}$ when $\mathbf{J}=\mathbf{K},(\mathbf{J}=\mathbf{2 4 , 2 5})$, but $[\ddot{E}(20)]$ yielded better test set classification performance than $\left[\ddot{E}_{t m, \max } \mathrm{J}(5 \times 4)\right](\mathbf{J}=\mathbf{2 4 , 2 5})$ for either of the 1-propanol/2-propanol mixture classification tasks $\mathbf{J}=\mathbf{2 2}$, 23. Interestingly, any [ $\left.\ddot{E}_{\mathrm{trn}, \max } \mathbf{J}(5 \times 4)\right](\mathbf{J}=\mathbf{2 2 - 2 7})$ array except $\left[\ddot{\mathrm{E}} \mathrm{trn}, \max { }^{25}(5 \times 4)\right]$ yielded a better test set classification performance for the $\mathrm{m}$-xylene/p-xylene mixture classification tasks, $\mathbf{J}=\mathbf{2 6}, \mathbf{2 7}$, than was obtained using the full 20 compositionally different Set B detector array, [Ë(20)]. The relatively low test set 
classification performance obtained when using the entire 20 detector Set B array on tasks 26 and 27 suggests that the $m$-xylene/ $p$-xylene separation is impeded by some detectors that have particularly low signal/noise ratios for this specific classification task.

Table 5. Classification Performance of Arrays Formed from Four Copies of Five Compositionally Different Detectors

\begin{tabular}{|c|c|c|c|c|c|c|c|}
\hline \multirow[t]{2}{*}{ Array: } & \multicolumn{7}{|c|}{ Task: } \\
\hline & & 22 & 23 & 24 & 25 & 26 & 27 \\
\hline Designation & Detectors & \multicolumn{5}{|c|}{ Absolute Performance } & \\
\hline$\left[\ddot{E}_{t \mathrm{tr}, \max }^{22}(5 \times 4)\right]$ & $8,9,11,18,19$ & 0.815 & 0.830 & 0.875 & 0.925 & 0.915 & 0.935 \\
\hline$\left[\ddot{E}_{\mathrm{trn}, \max }^{23}(5 \times 4)\right]$ & $8,11,13,18,19$ & 0.805 & 0.815 & \multicolumn{2}{|c|}{0.8250 .910} & 0.875 & 0.945 \\
\hline$\left[\ddot{E}_{t r n, \max }{ }^{24}(5 x 4)\right]$ & $1,8,15,19,20$ & 0.690 & 0.640 & 0.965 & 0.975 & 0.895 & 0.925 \\
\hline$\left[\ddot{E}_{\mathrm{trn}, \max }^{25}(5 \mathrm{x} 4)\right]$ & $1,3,4,19,20$ & 0.725 & 0.640 & 0.925 & 0.955 & 0.675 & 0.820 \\
\hline$\left[\ddot{E}_{\mathrm{trn}, \max }^{26}(5 \mathrm{x} 4)\right]$ & $4,8,9,12,18$ & 0.700 & 0.705 & 0.845 & 0.780 & 0.880 & 0.920 \\
\hline$\left[\ddot{E}_{\mathrm{trn}, \max }^{27}(5 \times 4)\right]$ & $1,8,9,12,19$ & 0.675 & 0.575 & 0.885 & 0.885 & 0.865 & 0.885 \\
\hline [Ё (20)] & & 0.755 & 0.755 & 0.920 & 0.930 & 0.810 & 0.845 \\
\hline Designation & \multicolumn{7}{|c|}{ Normalized Performance $^{a}$} \\
\hline$\left[\ddot{E}_{t r n, m a x} 22(5 x 4)\right]$ & & 1.24 & 1.29 & 0.893 & 0.988 & 1.34 & 1.26 \\
\hline$\left[\ddot{E}_{t r n, \max } 23(5 x 4)\right]$ & & 1.20 & 1.24 & 0.774 & 0.953 & 1.21 & 1.29 \\
\hline$\left[\ddot{E}_{\mathrm{tm}, \max }{ }^{24}(5 \times 4)\right]$ & & 0.745 & 0.549 & 1.11 & 1.10 & 1.27 & 1.23 \\
\hline$\left[\ddot{E}_{t m, m a x}{ }^{25}(5 \times 4)\right]$ & & 0.882 & 0.549 & 1.01 & 1.06 & 0.565 & 0.928 \\
\hline$\left[\ddot{E}_{t r n, \max }{ }^{26}(5 \times 4)\right]$ & & 0.784 & 0.804 & 0.821 & 0.651 & 1.23 & 1.22 \\
\hline$\left[\ddot{E}_{\mathrm{trn}, \max }^{27}(5 \times 4)\right]$ & & 0.686 & 0.294 & 0.917 & 0.895 & 1.18 & 1.12 \\
\hline
\end{tabular}

${ }^{a}$ Prediction abilities for each task are normalized, with 1.00 representing a task being applied to all 20 sensors. Normalization was accomplished from the formula Value $_{\text {norm }}=\left(\right.$ Value $\left._{\text {raw }}-0.5\right) /($ Norm- 0.5$)$ where "Norm" represents the number by which Value raw $_{\text {is }}$ to be normalized.

\subsection{Discrimination Performance Between Benzene and Toluene and Between 1-Propanol and 2- Propanol as a Function of Analyte Concentration}

The vapor classification tasks discussed above involved differentiation, at relatively high signal/noise ratios, between analytes that are so chemically similar that the signals produced by the detector arrays of interest did not result in well-separated clusters for the various analytes in d-dimensional detector response space. A conceptually different challenge for an array of semi-selective vapor detectors involves classification between analytes at sufficiently vapor phase concentrations that the low corresponding signal/noise responses for many of the detectors in the array will reduce the separation between data clusters that are otherwise wellseparated at high analyte concentrations. To probe the effects of array size and array composition on such tasks, detector response data were collected for conducting polymer composites that were exposed to benzene, toluene, 1-propanol, and 2-propanol, respectively, each at both high and low vapor phase concentrations (tasks 28-29, Table 2).

The 5-detector arrays that yielded the maximum Fisher RF value on training set data, [Ë ${ }_{t m, \max }{ }^{J}(5)$ ], for each classification task in the range $\mathbf{J}=\mathbf{2 8 - 2 9}$ were again selected from the collection of 20 compositionally different Set B detectors, [Ё (20)]. Table 6 indicates the detectors in these [Ё trn,max $\left.^{J}(5)\right]$ arrays and presents the test set classification performance of these arrays on tasks 28-29. Table 6 clearly shows that different detectors formed the best-performing 5-detector arrays for different analyte classification tasks.

The compositional differences between these optimally performing 5-detector [ $\left.\ddot{E}_{t \mathrm{~m}, \max } \mathrm{J}^{\mathrm{J}}(5)\right]$ arrays were investigated in more detail for tasks 28-29 of the Series II data run. To avoid any bias that might arise from incrementally small differences in classification performance between the best-performing detector set for a task, [Ë trn,max (5)], and other nearly optimal 5-detector sets for the same classification task, detectors were ranked based on the frequency with which a detector was contained in the 205 -detector arrays that produced the 20 best Fisher RF values on the training set data for the classification task of interest. Table 6 summarizes the results of such an analysis for tasks 28-31 of the Series II data. 
Table 6. Detectors Represented in Best-Performing 5-Detector Arrays for High Concentration and Low Concentration Mixture Classification Tasks

\begin{tabular}{|c|c|c|c|c|c|c|c|c|c|c|c|}
\hline & $\underline{T a s}$ & Detecto & elected & Most Fre & itly, $S$ & values, & ber of & imes Sele & & & \\
\hline 28 & 15 & 16.96 & 16 & $9.83 \quad 16$ & 18 & $186 \quad 20$ & 19 & 63.220 & 20 & 30.0 & 16 \\
\hline 29 & 1 & 13120 & 3 & 50.920 & 5 & 41.620 & 16 & 24.616 & 18 & 1238 & 4 \\
\hline \multicolumn{3}{|c|}{ Designation } & \multicolumn{6}{|c|}{ Best Overall Sets of Five Detectors ${ }^{\mathrm{a}}$} & \multicolumn{3}{|c|}{ Performance } \\
\hline \multirow{2}{*}{\multicolumn{3}{|c|}{$\left[\ddot{E} t \operatorname{trn}, \max _{29}^{28}(5)\right]$}} & 1 & 16 & 18 & 19 & \multicolumn{2}{|l|}{20} & \multicolumn{3}{|c|}{0.920} \\
\hline & & $\left.E_{t n, \max }(5)\right]$ & 1 & 3 & 5 & 10 & 16 & & \multicolumn{3}{|c|}{1.00} \\
\hline
\end{tabular}

For the low concentration 1-propanol vs. 2-propanol analyte classification task (task 28), the detectors that were most commonly contained in the 20 best-performing arrays (appearing in more than 10 out of the 20 array that yielded the best training set Fisher RF values) were detectors 16(17), 18(20), 19(20), and 20(16), with the numbers in parentheses indicating the number of the 20 total best-performing arrays for the task under consideration which contained that specific detector. Three of these detectors, 18, 19, and 20, were among the four detectors that exhibited the largest $\mathrm{S} / \mathrm{N}$ ratios for 1-proponol at $\mathrm{P} / \mathrm{P}^{\circ}=0.0075$, having $\mathrm{S} / \mathrm{N}$ values of 186 , 63.2 , and 30.0 , respectively. This makes sense in that a priority at low concentration conditions is to robustly detect the signal relative to the baseline detector noise, and robust detection will in general correlate with good analyte discrimination under such conditions.

In contrast, the detectors most frequently contained in the 205 -detector arrays that yielded the best Fisher RF training set values for the high concentration 1-propanol vs. 2-propanol analyte classification task (task 29) were detectors $\mathbf{1}(20), \mathbf{3}(20), \mathbf{5}(20)$, and 16(16). Only detector 1 had a relatively high $\mathrm{S} / \mathrm{N}$ value (131) for 1-propanol at $\mathrm{P} / \mathrm{P}^{0}=0.075$, with the other detectors exhibited $\mathrm{S} / \mathrm{N}$ ratios for 1-propanol at $\mathrm{P} / \mathrm{P}^{0}=0.075$ of 50.9, 41.6, and 24.6, respectively. Although these detectors were not among the most sensitive for the task at hand, they were selected among the 20 best-performing 5-detector arrays a total of 76 times, which is the maximum frequency with which four detectors could be selected among 20 unique sets of 5 detectors. Because this detector selection preference persisted despite the presence of detectors that possessed higher $\mathrm{S} / \mathrm{N}$ values for 1-propanol, such as detectors 8, 18, and 20 (S/N between 309 and 1240), the selected detectors are clearly providing more classification information between the two analytes in the test set under conditions when discrimination is more important than robust signal detection.

\section{CONCLUSIONS}

For a broad set of chemically very different test solvents, in principle only two semi-selective detectors are needed to provide robust information on the identity and concentration of any pure analyte in the test set. For carbon black composite chemiresistive vapor detectors, excellent classification performance was observed for arrays as small as 3-4 detectors for pure analyte vapors at concentrations high enough to produce high detector signal/noise ratios for the analytes of interest. However, when the signal strength was lowered, or when the analytes were chemically very similar, more detectors were required to achieve optimum classification performance for all tasks investigated. Classification performance in general either increased or did not decrease significantly as the number of chemically different detectors increased. Furthermore, different subsets of the detector array produced the best classification performance at a fixed array size for different analyte classification tasks. Hence, the full compositionally different detector array always yielded better classification performance than any smaller size array for at least some vapor classification tasks. Reduction in dimensionality was observed to be advantageous when the task was identified in advance, because multiple copies of detectors that had been identified as yielding the best test set classification performance for a given task at a fixed array size yielded better classification performance than the same total number of compositionally different detectors when both arrays were used for that specific classification task. However, the full array of compositionally different detectors yielded better test set classification performance than did any fixed array containing multiple copies of fewer compositionally different detectors for at least some other task of interest in a broadly construed set of analyte classification tasks. Subsets of detectors were identified that yielded robust discrimination between $\mathrm{D}_{2} \mathrm{O}$ and $\mathrm{H}_{2} \mathrm{O}$, between compositionally similar mixtures of 1propanol and 2-propanol, and between compositionally similar mixtures of $n$-hexane and $n$-heptane, attesting to the excellent analyte discrimination power that can be obtained at least in certain tasks through use an array of semi-selective chemiresistive vapor detectors even when no single detector provides the needed chemical resolution to differentiate between the analytes of interest. 


\section{ACKNOWLEDGMENTS}

We acknowledge the NIH and an Army MURI for their generous support of this work.

\section{REFERENCES}

(1) Gardner, J. W.; Bartlett, P. N. Electronic Noses: Principles and Applications; Oxford Science Publications: Oxford, 1999.

(2) Albert, K. J.; Lewis, N. S.; Schauer, C. L.; Sotzing, G. A.; Stitzel, S. E.; Vaid, T. P.; Walt, D. R. Chem. Rev. 2000, 100, 2595-2626.

(3) Patel, S. V.; Jenkins, M. W.; Hughes, R. C.; Yelton, W. G.; Ricco, A. J. Anal. Chem. 2000, 72, 15321542.

(4) Park, J. M.; Groves, W. A.; Zellers, E. T. Analytical Chemistry 1999, 71, 3877-3886.

(5) Doleman, B. J.; Lonergan, M. C.; Severin, E. J.; Vaid, T. P.; Lewis, N. S. Anal. Chem. 1998, 70, 41774190.

(6) Zellers, E. T.; Batterman, S. A.; Han, M.; Patrash, S. J. Anal. Chem. 1995, 67, 1092-1106.

(7) Zellers, E. T.; Park, J.; Hsu, T.; Groves, W. A. Analytical Chemistry 1998, 70, 4191-4201.

(8) Rose-Pehrsson, S.; Grate, J.; Ballantine, D. S.; Jurs, P. C. Anal. Chem. 1988, 60, 2801-2811.

(9) Grate, J. W.; Abraham, M. H. Sens. Actuators, B 1991, 3, 85-111.

(10) Grate, J. W.; Martin, S. J.; White, R. M. Anal. Chem. 1993, 65, 940A-948A.

(11) Hierlemann, A.; Weimar, U.; Kraus, G.; Schweizer-Berberich, M.; Gopel, W. Sens. Actuators, B 1995, 26-27, 126-134.

(12) Nakamoto, T.; Fukunishi, K.; Moriizumi, T. Sens. Actuators B 1990, 1, 473-476.

(13) Hatfield, J.; Neaves, P.; Hicks, P. J.; Persaud, K.; Travers, P. Sens. Actuators, B 1994, 18-19, $221-228$.

(14) Hodgins, D. Sensors and Actuators B. 1995, 27, 255-258.

(15) Dickinson, T. A.; Michael, K. L.; Kauer, J. S.; Walt, D. R. Anal. Chem. 2000, 71, 2192-2198.

(16) Albert, K. J.; Walt, D. R. Anal. Chem. 2001, 73, 2501-2508.

(17) Ronot, C. Sens. Actuators, B 1993, 11, 375-381.

(18) Sutter, J. M.; Jurs, P. C. Anal. Chem. 1997, 69, 856-862.

(19) Lonergan, M. C.; Severin, E. J.; Doleman, B. J.; Beaber, S. A.; Grubbs, R. H.; Lewis, N. S. Chem. Mater. 1996, 8, 2298-2312.

(20) Doleman, B. J.; Sanner, R. D.; Severin, E. J.; Grubbs, R. H.; Lewis, N. S. Anal. Chem. 1998, 70, 25602564.

(21) Duda, R. O.; Hart, P. E. Pattern Classification and Scene Analysis; John Wiley \& Sons: New York, 1973.

(22) Vaid, T. P.; Burl, M. C.; Lewis, N. S. Anal. Chem. 2001, 73, 321-331.

(23) Severin, E. J.; Doleman, B. J.; Lewis, N. S. Anal. Chem. 2000, 72, 658-668.

(24) Severin, E. J.; Lewis, N. S. Anal. Chem. 2000, 72, 2008-2015. 